Proceedings of the 1999 IEEE

International Conference on Robotics \& Automation

Detroit, Michigan • May 1999

\title{
A Task-Dependent Approach to Minimum-Deflection Fixtures
}

\author{
Qiao Lin and Joel W. Burdick \\ Division of Engineering and Applied Science \\ California Institute of Technology \\ Pasadena, CA 91125
}

\begin{abstract}
This paper presents an approach to planning minimum-deflection fixtures for tasks whose characteristics are well understood. Based on an accurately deflned notion of deflection, we define a quality measure that characterizes the workpiece's deflection with respect to a set of external wrenches determined by the tasks. A novel scheme is proposed to model task wrenches, which can be used for practical manufacturing operations. This task modelling scheme is then used to obtain a convenient formulation of the task-dependent quality measure, which allows the quality measure to be efficiently computed. An example is presented to show that our approach can be effectively employed for planning compliant fixtures that are best suited to specified tasks.
\end{abstract}

\section{Introduction}

Compliance can play a significant role in fixturing. Due to the presence of compliance, a fixtured object (workpiece) will deform when subjected to external loads. Intuitively speaking, the object's "deflection" can be thought of as a scalar-valued function that characterizes the size of the displacements of the object's particles under the applied load. In applications such as part machining and assembly insertion, the magnitude of this deflection will limit the accuracy of the overall process for which the fixture was designed. This paper presents an approach to the minimum-deflection fixturing of workpieces. Since fixtures are often designed for specified tasks, our approach is based on a novel taskmodelling scheme and a quality measure designed for compliant fixtures. The task-modelling scheme is efficient and can be used to realistically represent a large class of manufacturing processes. The quality measure takes account of the characteristics of given tasks, is valid for general 2D and 3D fixtures employing any number of fixture elements (fixels), and holds for any reasonable compliance model. Using this quality measure, the optimal fixel placements that minimize the object's deformation can be determined. While we focus on fixtures, our approach may also be applied to grasps which work in a known environment.

The subject of this paper is related to two research areas: modelling of compliant grasps/fixtures and quality measures that assess grasp/fixture effectiveness. Compliance effects on fixtures and grasps have been investigated using linear and nonlinear contact models. The use of a linear spring compliance model was initiated by Hanafusa and Asada [5], and was subsequently extended by Nguyen [17], Howard and Kumar [6], Donoghue et al. [3]. Recognizing that the linear contact model is not theoretically or experimentally justified, Lin, Burdick and Rimon [10] used overlap functions to compute and analyze the grasp stiffness matrix for various nonlinear contact models, including the well-justified Hertz model. While we use the overlap-based approach for our examples, the approach applies to other compliance models as well.

A quality measure quantifies the effectiveness of fixtures or grasps. Prior research on quality measures has mostly focused on rigid grasps. Li and Sastry [8], Kirkpatrick et al. [7], and Ferrari and Canny [4] defined quality measures based on the worst-case rejection of disturbing wrenches comprising the entire wrench space. These quality measures assume different values depending on different choices of reference frames. This difficulty was circumvented by Markenscoff and Papadimitriou [13], and Mirtich and Canny [14], who focused on subsets of the wrench space. Teichmann [20] also addressed the frame-dependence problem by considering the worst-case frame choices. The quality measure used in this work derives its frame-independence from the way in which object deflections are assessed. Based on the quasi-rigid body assumption, such deflections are computed from pseudo-norms of rigid body velocities. We use the frame-invariant pseudo-norms reported in Ref. [9]; hence the resulting quality measure is frameinvariant.

The quantification of compliant fixture effectiveness has been conducted by the current authors for the case of quasi-rigid compliant bodies. Lin, Burdick and Rimon [11] identified characteristic parameters (the "principal stiffnesses") that could be used to define a frameinvariant quality measure. Recognizing the importance of deflections on the accuracy of manufacturing processes, Lin, Burdick and Rimon [12] defined a quality measure as the fixtured object's worst-case deflection with respect to wrenches lying in a well-defined ball. These methods generate optimal fixture plans in the case where the task is not well understood. However, fixtures are often designed for specified tasks in highly structured environments. It is therefore highly desirable for fixture planners to account for the characteristics of such tasks. Task-dependent planning of rigid grasps 
or fixtures has been considered by Brost and Peters [2], who evaluated fixture effectiveness using the fixels' maximal reaction force to given tasks, and by $\mathrm{Li}$ and Sastry [8]. The latter authors used "task ellipsoids" to model grasping tasks, and defined a quality measure as the radius of the largest task ellipsoid that can be embedded in the set of wrenches resistible by finger forces up to a given magnitude. Rather than rigid fixtures, this paper is intended to address task-dependent planning of compliant fixtures.

The paper is organized as follows. Section 2 reviews c-space based modelling of compliant fixtures. Section 3 defines a task-dependent fixture quality measure, and Section 4 describes a novel task-modelling scheme. The computation of the quality measure based on this task model is considered in Section 5 .

\section{C-Space Based Compliance Modelling}

When contacting bodies are quasi-rigid, compliant fixtures can be studied using rigid body kinematics. Before describing the quasi-rigid body assumption, we briefly review the configuration space of a rigid body.

\subsection{Configuration Space Representation}

Let $\mathcal{B}$ be a rigid body in a $3 \mathrm{D}$ (or 2D) space. Let $\mathcal{F}_{W}$ be a stationary world reference frame, and $\mathcal{F}_{B}$ a frame fixed to $\mathcal{B}$. A configuration of $\mathcal{B}$ is a pair $q=$ $(p, R)$, where $p \in \mathbb{R}^{3}$ is the position, and $R \in S O(3)$ the orientation of $\mathcal{F}_{B}$ relative to $\mathcal{F}_{W}$. The set of all configurations, denoted $\mathcal{C}$, is $\mathcal{B}$ 's configuration space (cspace). The tangent space to $\mathcal{C}$ at configuration $q$, is the set of all tangent vectors (velocities) of $\mathcal{B}$ at $q$. Tangent vectors can be viewed as an instantaneous displacements of $\mathcal{B}$ and can approximate small displacements. The wrench space at $q$ is the set of all wrenches, or covectors, acting on $\mathcal{B}$ at configuration $q$.

The body coordinates [15] of tangent vectors and covectors are defined as follows. A tangent vector is specified as a body velocity in the form $\dot{q}=(v, \omega) \in \mathbb{R}^{3} \times \mathbb{R}^{3}$, where $v$ is the velocity of $\mathcal{F}_{B}$ 's origin and $\omega$ is $\mathcal{F}_{B}$ 's angular velocity, as seen by an observer in $\mathcal{F}_{B}$. A covector is specified as a body wrench, $\boldsymbol{w}=(f, \tau) \in \mathbb{R}^{3} \times \mathbb{R}^{3}$, where $f \in \mathbb{R}^{3}$ is a force acting at $\mathcal{F}_{B}$ 's origin and $\tau$ is a torque. A more commonly used parametrization is based on hybrid velocities, $\dot{q}_{h}=\left(v_{h}, \omega_{h}\right) \in \mathbb{R}^{3} \times \mathbb{R}^{3}$, and hybrid wrenches, $\boldsymbol{w}_{h}=\left(f_{h}, \tau_{h}\right)$. The vectors $v_{h}$ and $\omega_{h}$, and $f_{h}$ and $w_{h}$ have the same meanings as their counterparts in the body coordinates $\dot{q}$ and $\boldsymbol{w}$, but are specified with respect to $\mathcal{F}_{W}$. Thus, if $R$ is the orientation of $\mathcal{F}_{B}$ relative to $\mathcal{F}_{W}$, then the two representations are related by $\dot{q}_{h}=\mathcal{R} \dot{q}$ and $w_{h}=\mathcal{R} w$, where $\mathcal{R}=\operatorname{diag}(R, R)$. Since these rules can be used to transform any expression in body coordinates into one in hybrid coordinates, we use body velocities and wrenches in this paper.
Since body coordinates are specified with respect to the body frame, it is clear that they are not affected by change of world frame. The change-of-body-frame formulas for body velocities and wrenches are given by [15]

$$
\dot{\bar{q}}=T_{g}^{-1} \dot{q} \quad \text { and } \quad \overline{\boldsymbol{w}}=T_{g}^{\tau} \boldsymbol{w} \text {. }
$$

where overbars denote objects with respect to a new body frame, denoted $\overline{\mathcal{F}}_{B}$, which is displaced from $\mathcal{F}_{B}$ by a rigid displacement $g=(d, R)$. The matrix $T_{g}$ takes the form $T_{g}=\left(\begin{array}{c}R \widehat{d} R \\ 0\end{array}\right)$, where for a given $x \in \mathbb{R}^{3}, \widehat{x}$ is a skew-symmetric matrix such that $\widehat{x} y=x \times y$ for $y \in \mathbb{R}^{3}$.

\subsection{Modelling of Compliant Fixtures}

A fixture consists of an object (or workpiece), denoted $\mathcal{B}$, contacted by $k$ fixture elements (or fixels), denoted $\mathcal{A}_{1}, \ldots, \mathcal{A}_{k}$. We make the following assumptions for our study. First, the bodies $\mathcal{B}$ and $\mathcal{A}_{i}$ are quasi-rigid, i.e., the compliant deformations are restricted to the vicinity of the contacts. Second, each fixel is stationary, and we can hence focus on $\mathcal{B}$ 's c-space, $\mathcal{C}$. Finally, the fixture is a conservative elastic system whose elastic behavior can be characterized by a scalar-valued function, called the elastic potential and denoted $\Pi(q)$.

A configuration $q_{0}$ is said to be an equilibrium configuration if $\mathcal{B}$ is in equilibrium under nonzero fixel forces in the absence of external disturbances. We call the nonzero fixel forces preloading forces and the fixture a preloaded equilibrium fixture. An equilibrium configuration $q_{0}$ is a critical point of $\Pi$, that is, $\nabla \Pi\left(q_{0}\right)=0$. The $6 \times 6$ Hessian matrix, $K=D^{2} \Pi\left(q_{0}\right)$, is the equilibrium fixture stiffness matrix. As an elastic system, the fixture at $q_{0}$ is (quasi-statically) stable if $q_{0}$ is a local minimum of $\Pi$. The fixture is stable if $K$ is positive definite. In this case, letting $\dot{q}$ be a body velocity used to approximate the small displacement of $\mathcal{B}$ due to a disturbing body wrench $w$, we have the linear relationship: $w=K \dot{q}$ or $\dot{q}=C w$, where $C=K^{-1}$ is called the fixture's compliance matrix.

The elastic potential $\Pi(q)$ can be obtained from a contact model, which correlates fixel forces to relative displacements between the object and fixels. The work reported in this paper is general in the sense that it is applicable to all contact models that are consistent with the quasi-rigidity assumption. We may use the modelling scheme that is based on virtual overlaps of quasirigid bodies [19], which is briefly reviewed here. We wish to ignore the details of compliant surface deformations due to a relative displacement of the contacting bodies, and model the resultant contact force as a function of $\mathcal{B}$ 's relative displacement. Instead of solving for the complex surface deformations that arise during compliant interaction, imagine that the rigid shape of $\mathcal{B}$ freely penetrates the rigid shape of $\mathcal{A}_{i}$ during the relative approach that accompanies their true compression. I.e., the two quasi-rigid bodies virtually "overlap" as their 
bodies are pressed together. The virtual overlap between $\mathcal{B}$ and $\mathcal{A}_{i}$ can be characterized by a line segment, of length $\delta_{i}(q)$, which lies in the overlapping region with endpoints on the boundaries of $\mathcal{B}$ and $\mathcal{A}_{i}$. Note that the length of the overlap segment depends on $\mathcal{B}$ 's configuration $q$ (since $\mathcal{A}_{i}$ is stationary). Using this segment as a lumped-parameter representation of elastic deformations at the contact, the resultant force on $\mathcal{B}$ applied by the fixel $\mathcal{A}_{i}$ can be written as a function $f_{i}\left(\delta_{i}(q)\right)$. This allows us to obtain an expression for $\Pi(q)$, and compute the stiffness matrix. As discussed in Ref. [10], this approach can be used to incorporate a variety of contact models, including the well-justified Hertz contact model.

The stiffness of a preloaded fixture increases with the preloading forces. However, the preloading must be within the limit of contacting bodies' allowable strength. The appropriate preloading level can be determined from the chosen compliance model. For concreteness the following discussion uses the Hertz contact model that is expressed using overlap functions. The maximum shear stress [21] in the contacting bodies can be expressed as a function of the overlap: $\tau_{\max i}\left(\delta_{i}(q)\right)$. From theory of strength of materials [21], if $\tau_{\max i} \leqslant \sigma_{y}$, where $\sigma_{y}$ is the bodies' yield stress, then the bodies remain elastic. Let $q_{0}$ be an equilibrium configuration of a $k$ fixel fixture. Then we determine the preloading level by $\max _{1 \leqslant i \leqslant k} \tau_{\text {maxi }}\left(\delta_{i}\left(q_{0}\right)\right)=\zeta \sigma_{y}$, where $0<\zeta<1$ is a constant. In other words, the fixture is preloaded to a certain percentage of the materials' allowable strength. After determining the maximum shear stress, we can find the individual preloading fixel forces and the preloading overlaps [9].

In fixture planning, it is also important to ensure that there is no contact breakage resulting from the fixtured object's displacement, and that there is no excessive deformation beyond the bodies' material strength. These requirements, called the contact maintainability conditions [9], can be expressed as algebraic constraint inequalities that must be satisfied by a proposed fixture plan. In the context of the overlap-based compliance modelling scheme, this condition can be expressed as $0 \leqslant \delta_{i}\left(q_{0}+\dot{q}\right) \leqslant \delta_{i_{\max }}$, where $\dot{q}$ is the displacement of $\mathcal{B}, q_{0}$ is configuration of $\mathcal{B}$ prior to the displacement, and $\delta_{i_{\max }}$ is the maximally allowed overlap dictated by material strength.

\section{A Task-Dependent Quality Measure}

This section defines a task-dependent quality measure that characterizes the deflection of a fixtured object induced by task execution. We only consider stable fixtures with positive definite stiffness matrices, since other types of fixtures are considered ineffective.

We first formally introduce the notion of deflection.
Let a quasi-rigid workpiece, denoted $\mathcal{B}$, undergo a displacement approximated by a body velocity. To ensure the accuracy of task execution, it is often essential to monitor the corresponding displacements of $\mathcal{B}$ 's particles. The notion of deflection is defined for this purpose.

Definition 1. A deflection of $\mathcal{B}$ corresponding to a displacement $\dot{q}$ is a scalar-valued function $\varphi(\dot{q})$ with the following properties. First, $\varphi(\dot{q}) \geqslant 0$ for any $\dot{q}$ (i.e., $\varphi$ is non-negative). Second, $\varphi(\alpha \dot{q})=|\alpha| \varphi(\dot{q})$ for any scalar $\alpha$ (i.e., $\varphi$ scales linearly with the displacement). Third, $\varphi\left(\dot{q}_{1}+\dot{q}_{2}\right) \leqslant \varphi\left(\dot{q}_{1}\right)+\varphi\left(\dot{q}_{2}\right)$ for any $\dot{q}_{1}$ and $\dot{q}_{2}$ (i.e., $\varphi$ satisfies the triangle inequality). Finally, $\varphi$ transforms in a frame-invariant manner, i.e., $\varphi(\dot{q})=\bar{\varphi}(\dot{\bar{q}})$, where overbars denote objects in a new body frame.

In the above definition, only body-frame changes must be considered, since body velocities are not affected by the choice of world frame. Also, we did not require $\varphi(\dot{q})$ to be positive definite for the following reason. We often need to monitor the displacements of only a finite collection of important workpiece points, such as the vertices of polyhedral objects or key features in the interior of an object. As $\mathcal{B}$ experiences a nonzero displacement, the important points may remain at their original positions. This may occur when there are very few important feature points (e.g less than 2 in the 2D case). In mathematical terms, we recognize the function $\varphi$ as a pseudo-norm [16] of rigid body velocities.

We now give a specific deflection function which will be used to define our task-dependent quality measure. Denote by $\Omega$ the set of critical points of $\mathcal{B}$, which is assumed to be finite. There is little loss of generality in the assumption. Let $\mathcal{B}$ undergo a displacement approximated by $\dot{q}=(v, \omega)$, a body velocity. Then the function defined below is a deflection function [9]:

$$
\varphi(\dot{q})=\max _{r \in \Omega}\|v+\omega \times r\| .
$$

Since $u_{r}=v+\omega \times r$ is the displacement of $r \in \Omega$ corresponding to $\mathcal{B}$ 's movement by $\dot{q}$, the deflection function gives the maximal displacement of the points lying in $\Omega$. This physical interpretation makes $\varphi$ well-suited for workpiece fixturing, where the critical points' maximal displacement often has a direct impact on the precision of task execution.

While the deflection defined in (2) is used in this paper, we note that the definition of deflection is not unique. For example, we may define the deflection to be the root mean square of the displacements of the points in $\Omega$. This deflection function was used in Ref. [12].

We now define a fixture quality measure that characterizes the object's deflection with respect to the tasks for which the fixture is designed. The task's influence on the fixture is modeled by a task wrench set, $W$, which we assume to be a compact subset of the wrench space. Under the action of an external wrench $\boldsymbol{w} \in W$, the 
fixtured workpiece undergoes an approximate displacement $\dot{q}=C w$, where $C$ is the fixture's compliance matrix, and the grasp is assumed to be stable. Since the task wrench set is compact, $\varphi(C \boldsymbol{w})$ is bounded. Hence, we can define the following quality measure:

$$
Q=\max _{\boldsymbol{w} \in W} \varphi(C w)
$$

We observe that $Q$ determines the worst-case deflection of the points in $\Omega$ with respect to all wrenches in the task wrench set. A key issue is to develop tractable computations for Eq. (3) for practical cases.

When calculating the deflection according to (2), the displacement of $r \in \Omega$ corresponding to a rigid displacement $\dot{q}$ of $\mathcal{B}$ can be written as $u_{r}=D_{r} \dot{q}$ where $D_{r}=[-\widehat{r} I]$. It follows that the deflection is given by $\varphi(C w)=\max _{r \in \Omega}\left\|u_{r}(\boldsymbol{w})\right\|$, where $u_{r}(\boldsymbol{w})=D_{r} C \boldsymbol{w}$ is the displacement of the point $r$ when $\mathcal{B}$ is under the action of the wrench $w$. Hence, we can rewrite (3) as

$$
Q=\max _{\boldsymbol{w} \in W} \max _{r \in \Omega}\left\|u_{r}(\boldsymbol{w})\right\|=\max _{r \in \Omega} \max _{\boldsymbol{w} \in W}\left\|u_{r}(\boldsymbol{w})\right\| .
$$

Since $\Omega$ contains only a finite number of points, we can for each $r \in \Omega$ focus on $\max _{\boldsymbol{w} \in W}\left\|u_{r}(\boldsymbol{w})\right\|$, which is the maximal displacement of $r$ with respect to all wrenches in the task wrench set $W$.

\section{Modelling of Task Wrench Sets}

This section describes a novel scheme for characterizing task wrench sets appropriate to workpiece fixturing. First observe that during task execution, the task wrench may be difficult to predict accurately. For example, in the milling operation illustrated in Fig. 1, the cutter exerts a force on the workpiece. While the magnitude of the cutting force can be estimated from machining power, the direction of the force is difficult to determine and may additionally vary to due vibrations, etc. Thus, the force may be assumed to lie in a sector (shown in the figure), which moves along the cutting path with the cutter. Brost and Peters [2] suggested the use of a discrete set of positions and directions to approximate the continuous task data. However, this approach can be computationally expensive for 3D fixtures and it does not accurately account for all wrenches generated during continuous cutter motion. Therefore, it is desirable to develop a procedure for conveniently modelling such continuously evolving task wrench sets. The modelling of tasks for robotic grasping was considered by Li and Sastry [8] using ellipsoids in the wrench space. We propose a modelling scheme that is more appropriate for workpiece fixturing on the basis of the following observations.

To build some intuition for our modelling scheme, consider some common operations that involve fixtures. First consider assembly fixtures, which are also known as assembly pallets. As a fixtured part hits a motion

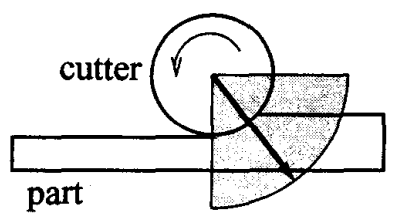

Fig. 1. Cutting force in a milling operation

stop during its transfer from station to station, it will be quickly decelerated. Due to this deceleration, the part is subjected to an inertial force acting at the part's center of mass. Thus the task wrench set is simply a finite collection of known wrenches.

Next consider fixtures for machining purposes. As shown in the milling example given above, machining operations exert a wrench (i.e., a force and a torque) on the workpiece. Due to uncertainties, the wrench may not be completely known, and in general varies as the cutter moves. However, the component of the wrench consisting of forces can often be localized to a given range of directions, with known magnitude along each direction. That is, the force component lies in a given compact set that is independent of the location of the task wrench's application. Since the force components of the task wrenches all lie in a fixed set, the task wrench set is a subset of a cylinder (interior points included) in the wrench space based on the fixed force component set. For instance consider the milling operation shown in Fig. 1. As shown in Fig. 2(a), with respect to the frame $\overline{\mathcal{F}}_{B}$, the force domain is a sector given by

$$
F=\left\{(f \cos \phi, f \sin \phi):-\frac{\pi}{4} \leqslant \phi \leqslant \frac{\pi}{4}, 0 \leqslant f \leqslant \rho_{f}\right\} .
$$

This expression defines a cylinder in the $3 \mathrm{D}$ wrench space whose base set is $F$ and whose generators are parallel to the $\tau$-axis. The torque of such a force can be written as $\tau=\xi\left(f_{x} \sin \alpha-f_{y} \cos \alpha\right)$, where $\xi \in\left[\xi_{1}, \xi_{2}\right]$. Thus, the task wrench set is a subset of the cylinder bounded by two planes given by $\tau-\xi_{i}\left(f_{x} \sin \alpha-f_{y} \cos \alpha\right)=0$, as shown in Fig. 2(b). Note that even when the magnitude or direction range of the force component changes during the machining operation, one may conservatively assume such a cylindrical set provided the actual wrench set is a subset of the assumed set.

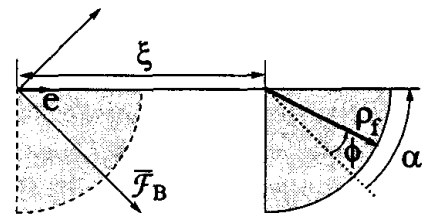

(a)

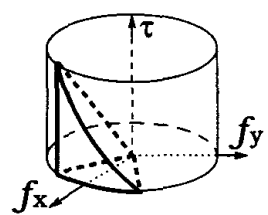

(b)
Fig. 2. Task primitive for milling operation

These observations suggest that we model task wrench sets as subsets of generalized cylinders in the wrench space, whose base sets are compact sets in the 
space of force components. We first introduce two types of primitive force domains. Let $\overrightarrow{\mathcal{F}}_{B}$ be a body frame. With respect to this frame, consider a sphere $S=\left\{f \in \mathbb{R}^{3}: f^{T} f \leqslant \rho_{f}^{2}\right\}$ and a half-space $H=\{f \in$ $\left.\mathbb{R}^{3}: n^{T} f \leqslant \rho_{h}\right\}$, where $n$ is a unit vector, and $\rho_{f} \geqslant 0$ and $\rho_{h} \in \mathbb{R}$ are constants. Note that the boundary of $H$ is normal to $n$. We define two types of force domains by considering the intersections of these sets with a circular cone given by $\Gamma=\left\{f \in \mathbb{R}^{3}: n^{T} f \geqslant\|f\| \cos \alpha\right\}$, which is symmetric about $n$ and has a vertical angle of $2 \alpha$ with $\alpha \in[0, \pi]$. The first type, called Type $I-3 D$, is defined by

$$
F=S \cap \Gamma,
$$

and the second type, called Type $I I-3 D$, by

$$
F=H \cap \Gamma \text {. }
$$

When $n=(0,0,1)$, i.e., $F$ is symmetric about the $f_{z^{-}}$ axis (Fig. 3), we say that $F$ is in canonical form. In body coordinates, $F$ is independent of the choice of world frame. Since any change of body frame may only result in a rigid rotation of $F$, the shape of $F$ remains the same for all frame choices.

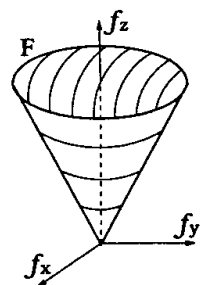

(a) Type I-3D

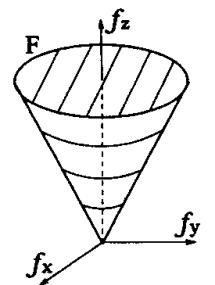

(b) Type II-3D
Fig. 3. Canonical forms of force domain

The I-3D and II-3D types of force domains can be used to represent force components of practical task wrenches. For example, in a drilling operation, the drill bit exerts a thrust force on the object. The direction of this force, which generally deviates from the specified drilling axis due to uncertainties, may be assumed to lie in a circular cone which is symmetric about the drilling axis. If one assumes that the thrust force has a magnitude estimated in a way independent of the force's direction, the thrust force can then be represented by a Type I-3D force domain. Type II-3D can be applied similarly in practice. This type can also be used to approximate Type I-3D force domains, since it involves no curved domain boundaries, and leads to considerable simplifications. See Fig. 6 for an analogy of the difference between Type I-3D and Type II-3D models.

We now present a general form for primitive wrench sets using body wrenches in the body frame $\overline{\mathcal{F}}_{B}$.

Definition 2. A primitive wrench set is a subset of the wrench space of the form

$\vec{W}=\left\{\left(f,(\Phi \xi) \times f+\Psi f+\tau_{0}\right) \in \mathbb{R}^{6}: f \in F, \xi \in P\right\}$, where $\Phi$ and $\Psi$ are appropriately dimensioned constant matrices, $\tau_{0}$ is a constant vector, $\xi \in P$ is a parameter vector, and the parameter domain $P$ is a compact polygonal subset of $\mathbb{R}^{2}$.

In this definition, we assume $P$ to be compact since cutter paths have finite dimensions, and $P \subset \mathbb{R}^{2}$ since cutting forces can only be applied on the workpiece's boundary. However, we do not make any assumptions on the convexity of $P$.

Intuitively, the proposed form of primitive wrench sets represent task wrenches that move along continuous paths. Moreover, we observe that the torque component of a primitive wrench in general consists of three terms. The first term accounts for the contribution of a moving pure force ( $\Phi \xi$ gives the position of $f$ ), the second term gives a torque that is related to the force component in some specified manner (see Example 2 below), and the third term is a constant torque. Specific examples of these terms can be found in Examples 1-4.

Geometrically, a primitive wrench set is a parametrized subset of a solid cylinder in the wrench space whose base set is $F$ and whose generators are orthogonal to $F$ and hence are pure torques. This is illustrated in Fig. 2(b), which shows the primitive wrench set for the milling operation of Fig. 1. The following two examples give some practically useful primitive wrench sets for $3 \mathrm{D}$ workpiece fixturing. Many other examples fit into our modelling framework.

Example 1. In Fig. 4 are shown two force cones that move over a polygonal region in space. The cones are both in canonical form with respect to the body frame $\overline{\mathcal{F}}_{B}$. The parameter domain is a planar region, specified with coordinate axes $\xi_{1}$ and $\xi_{2}$. Then this primitive wrench set is given by $\bar{W}=\left\{(f,(\Phi \xi) \times f) \in \mathbb{R}^{6}: f \in\right.$ $F, \xi \in P\}$, where $\Phi=\left[e_{1} e_{2}\right]$ with $e_{1}$ and $e_{2}$ being unit vectors, specified in $\overline{\mathcal{F}}_{B}$, along the $\xi_{1}$ and $\xi_{2}$ axes.

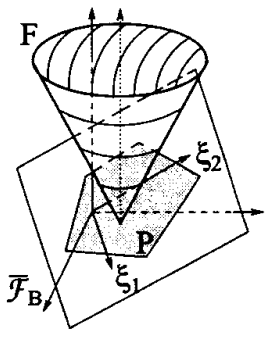

(a)

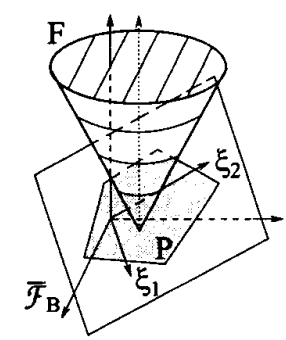

(b)
Fig. 4. Force cones moving over a polygon

Example 2. Fig. 5 shows a model of drilling operations. In this model a force of constant magnitude $\rho_{f}$ and a torque of constant magnitude $\rho_{\tau}$ are aligned along a line $\ell$, which lies in a cone as shown. We see that the base set $F$ is of Type I-3D, and that $\bar{W}=\left\{\left(f,\left(\rho_{\tau} / \rho_{f}\right) f\right) \in \mathbb{R}^{6}: f \in F\right\}$.

Now let us consider the special case of planar fix- 


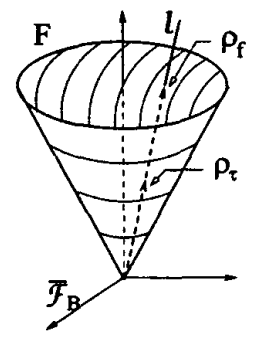

Fig. 5. A model for drilling operations

tures. The wrench space is 3-dimensional with scalar torque components. The parameter domain $P$ for primitive wrench sets is now a line segment. The Type I-3D force domain reduces to Type $1-2 D$ (the intersection of a disc with a planar cone), and Type II-3D reduces to Type II-2D (the intersection of a half-plane with a planar cone). Such force domains can be used to model the effect of 3D machining operations on an object that is restrained to move only in a plane (see Example 4), or machining (e.g., milling) operations that mainly generate $2 \mathrm{D}$ forces. For $2 \mathrm{D}$ force domains, we choose their canonical forms such that they are symmetric about the $f_{x}$-axis, as shown in Fig. 6 .

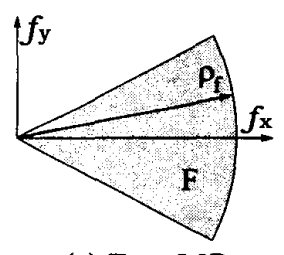

(a) Type I-2D

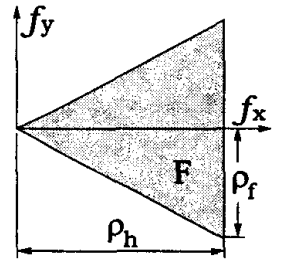

(b) Type II-2D
Fig. 6. Canonical forms of $2 \mathrm{D}$ force domain

Example 3. As shown in Fig. 7, two task primitives are given where a force sector and a force cone, both in canonical forms, move along a line segment $P$, respectively. Then in the frame $\overline{\mathcal{F}}_{B}$, the primitive wrench sets are of the form $\bar{W}=\left\{(f, \xi e \times f) \in \mathbb{R}^{3}: f \in F, \xi \in P\right\}$, where $e$ is a unit vector along $P$.

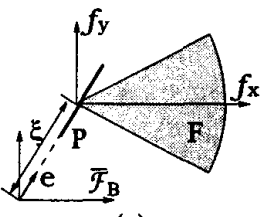

(a)

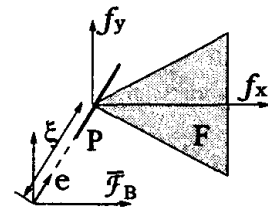

(b)
Fig. 7. Planar force cones moving along a line segment

Example 4. Fig. 8 shows a model of drilling operations for planar fixtures. This primitive wrench set takes the form $\bar{W}=\left\{\left(f, \tau_{d}\right) \in \mathbb{R}^{3}:\|f\| \leqslant \rho_{f}\right\}$.

\section{Task-Model Based Computation}

This section employs the primitive wrench sets, which were developed in Section 4, to compute the taskdependent quality measure. The verification of the con-

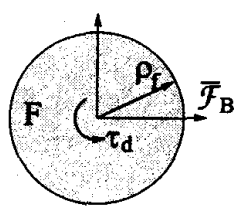

Fig. 8. A model for drilling operation in planar case

tact maintainability conditions can be performed in a similar manner and is discussed in Ref. [9].

\subsection{Formulation Using Primitive Wrench Sets}

We now consider the modelling of task wrench sets as composites of primitive sets. These composite sets can be used to represent quite general manufacturing operations. This approach allows us to formulate the taskdependent quality measure (3) using primitive wrench sets.

Consider a given task wrench set $W$. Let $\bar{W}_{1}, \ldots, \bar{W}_{m}$ be primitive wrench sets expressed in the intermediate body frames $\overline{\mathcal{F}}_{B 1}, \ldots, \overline{\mathcal{F}}_{B m}$ displaced from $\mathcal{F}_{B}$ by $g_{1}$, $\ldots, g_{m}$, respectively. We say that $W$ is modelled by $\vec{W}_{i}$ if for each $\boldsymbol{w} \in W$, there exist $\overline{\boldsymbol{w}}_{i} \in \bar{W}_{i}$ such that

$$
\boldsymbol{w}=\sum_{i=1}^{m} T_{g_{i}}^{-T} \overline{\boldsymbol{w}}_{i}
$$

where the wrench transformation rule in (1) has been used. Thus, the task wrench set is given by a primitive wrench set, or the Minkowski sum of several primitive wrench sets.

To compute the task-dependent quality measure, we can focus on computing $\max _{\boldsymbol{w} \in W}\left\|u_{r}(\boldsymbol{w})\right\|$, or equivalently, $\max _{\boldsymbol{w} \in W}\left\|u_{r}(\boldsymbol{w})\right\|^{2}$ for a particular point $r \in \Omega$ (Section 3). Here

$$
u_{r}(\boldsymbol{w})=D_{r} C \boldsymbol{w}
$$

is the displacement of the point $r$ due to the wrench $\boldsymbol{w}$, where $C$ is the fixture's compliance matrix, and $D_{r}=[-\hat{r} I]$. Let $\boldsymbol{w}$ be given in terms of primitive wrenches by (4), where each $\overline{\boldsymbol{w}}_{i}$ is determined by its force component $f_{i} \in F_{i}$ and parameter vector $\xi_{i} \in P_{i}$ (Section 4). The displacement $u_{r}(w)$ depends on $f=\left(f_{1}, \ldots, f_{m}\right) \in F=F_{1} \times \cdots \times F_{m}$ and $\left(\xi_{1}, \ldots, \xi_{m}\right) \in P=P_{1} \times \cdots \times P_{m}$, and by using the structure of primitive wrench sets, can be rewritten in the form [9]

$$
u_{r}(f, \xi)=\sum_{i=1}^{m} A_{i}\left(\xi_{i}\right) f_{i}+b(\xi)=\sum_{i=1}^{m} Z_{i}\left(f_{i}\right) \xi_{i}+y(f) \text {, }
$$

where $A_{i}\left(\xi_{i}\right), b(\xi), Z_{i}\left(f_{i}\right)$, and $y(f)$ are matrix- or vector-valued functions. It follows that $\left\|u_{r}\right\|^{2}$ is a convex function when either $f$ or $\xi$ is fixed. Since the maximum of a convex function is achieved at an $e x-$ treme point of the convex hull of its domain, $\left\|u_{r}(f, \xi)\right\|^{2}$ achieves its maximum at an extreme point of the convex hull of $P$ when $f$ is fixed. Now, $\max _{\boldsymbol{w} \in W}\left\|u_{r}(w)\right\|^{2}=$ $\max _{f \in F} \max _{\xi \in P}\left\|u_{r}(f, \xi)\right\|^{2}$. Thus, the global maximum 
of $\left\|u_{r}\right\|^{2}$ can be obtained by finding its maxima with respect to $f$ for each of the vertices of the convex hull of $P$. Let $\xi_{0}$ denote such a vertex, and consider $\tilde{u}(f) \triangleq u_{r}\left(f, \xi_{0}\right)$. Dropping for brevity the argument $\xi_{0}$ from $A_{i}$ and $b_{i}$, which are now constant, we can focus on $\max _{f \in F} \phi(f)$, where $\phi(f)=\|\tilde{u}(f)\|^{2}$, i.e.,

$$
\phi(f)=\left(\sum_{i=1}^{m} A_{i} f_{i}+b\right)^{T}\left(\sum_{i=1}^{m} A_{i} f_{i}+b\right) .
$$

Since $\phi(f)$ is a convex quadratic function, the maximizers of $\phi(f)$ belong to $\operatorname{ext}(F)=\operatorname{ext}\left(F_{1}\right) \times \cdots \times$ $\operatorname{ext}\left(F_{m}\right)$, where $\operatorname{ext}(F)$ and $\operatorname{ext}\left(F_{i}\right)$ are the set of extreme points of $F$ and $F_{i}$, respectively. Each $\operatorname{ext}\left(F_{i}\right)$ can be partitioned in the form $\operatorname{ext}\left(F_{i}\right)=\bar{F}_{i} \cup \widetilde{F}_{i}$, where $\bar{F}_{i}$ is a discrete set and $\widetilde{F}_{i}$ a continuous set. Suppose that $f_{1_{0}}$ is an element of $\bar{F}_{1}$. Then for maximizing $\phi(f)=\|\tilde{u}(f)\|^{2}$ over $\left\{f_{1_{0}}\right\} \times \widetilde{F}_{2} \times \cdots \times \widetilde{F}_{m}$, we may recast the function as $\tilde{u}\left(f_{2}, \ldots, f_{m}\right)=\sum_{i=2}^{m} A_{i} f_{i}+\left(A_{1} f_{1_{0}}+b\right)$. By reasoning along this line, we can ignore the discrete sets $\bar{F}_{i}$ without loss of generality and focus on maximizing $\phi(f)$ over $\widetilde{F}_{1} \times \cdots \times \widetilde{F}_{m}$. Now, we consider the typical form of the sets $\widetilde{F}_{i}$. Let $F_{i}$ be a primitive force domain described in Section 4 . Then $\widetilde{F}_{i}$ is either a circular arc or a spherical patch with a circular boundary, and is determined by the following two constraints:

$$
f_{i}^{T} f_{i}=\rho_{f_{i}}^{2} \text { and } n_{i}^{T} f_{i} \geqslant \rho_{f_{i}} \cos \alpha_{i}
$$

where all the quantities except $f_{i}$ are constant.

Therefore, while the quality measure (3) is a complicated function of wrenches that generally form a set of complex shape, the formulation described above allows us to focus on maximizing a positive definite quadratic function $\phi$ over the simple domain given by (6).

\subsection{Maximal Particle Displacements}

To find the maximal displacement of a particle of $\mathcal{B}$ with respect to a given task wrench set, we need to find the maximum of the function $\phi\left(f_{1}, \ldots, f_{m}\right)$, as defined in Eq. (5), over $\widetilde{F}_{1} \times \cdots \times \widetilde{F}_{m}$.

Since $\phi$ is a convex quadratic function, whose maximum over $\widetilde{F}_{i}$ determined by the constraints $(6)$ remains maximal over the region determined by $f_{i}^{T} f_{i} \leqslant \rho_{f_{i}}^{2}$ and $n_{i}^{T} f_{i} \geqslant \rho_{f_{i}} \cos \alpha_{i}$. Thus, we need to solve a convex quadratic maximization problem subjected to multiple convex quadratic constraints. Several algorithms have been developed for such optimization problems (e.g., [1]), and can be used to find the global maximum of $\phi(f)$.

We now discuss the following important special case where the task wrench set is modelled by a single task primitive. In this case, we wish to maximize

$$
\phi(f)=(A f+b)^{T} P(A f+b)
$$

over $\widetilde{F}$ determined by the constraints

$$
f^{T} f=\rho_{f}^{2} \text { and } n^{T} f \geqslant \rho_{f} \cos \alpha .
$$

The maximization problem affords a particularly simple solution when the force domain is planar $\left(f \in \mathbb{R}^{2}\right)$. The constraints in this case can be parameterized by $f=(\cos \theta, \sin \theta)^{T}$, where $\theta \in[-\alpha, \alpha]$. For maximizing $\phi(f)$, it is necessary that $(A f+b)^{T} \frac{\partial}{\partial \theta}(A f+b)=0$, i.e.,

$$
\left(-a_{1}^{T} \sin \theta+a_{2}^{T} \cos \theta\right)\left(a_{1} \cos \theta+a_{2} \sin \theta+b\right)=0,
$$

where $A=\left[\begin{array}{ll}a_{1} & a_{2}\end{array}\right]$. Substituting $\sin \theta=\left(1-t^{2}\right) /\left(1+t^{2}\right)$ and $\cos \theta=2 t /\left(1+t^{2}\right)$ yields a quartic equation in $t$, which can be solved to find the maximizer of $\phi$.

Now consider the case where $f \in \mathbb{R}^{3}$. If the maximizer of $\phi$ lies on the boundary of $\widetilde{F}$, i.e., the inequality constraint for $\widetilde{F}$ holds as an equality, then the technique for the 2D case can be invoked since $\widetilde{F}$ 's boundary is a circle. Thus, it remains to consider the case where $\phi$ is maximized at an interior point of $\widetilde{F}$ and the inequality constraint is inactive. Omitting the constant term, we can rewrite $\phi$ as

$$
\phi(f)=\frac{1}{2} f^{T} G f+d^{T} f,
$$

where $G=2 A^{T} A$ and $d=2 A^{T} b$. The global maximizer of $\phi$ over $\widetilde{F}$ is a local (but not necessarily global) maximizer of $\phi$ over the sphere $f^{T} f=\rho_{f}^{2}$. These local maximizers can be determined by the following stationarity conditions:

$$
(G-\mu I) f+d=0 \text { and } f^{T} f=\rho_{f}^{2},
$$

where $\mu>0$ is an undetermined Lagrange multiplier. By careful case studies considering the relationship between $\mu$ and the eigenvalues of the matrix $G$, these equations can be efficiently solved from polynomial equations of up to order 6 . The details of this solution process can be found in Ref. [9].

Thus, when the task wrench set can be modelled by a single wrench primitive, the quality measure (3) can be computed quite efficiently. This is of practical interest, since if a fixture is designed for a specified manufacturing operation (e.g., milling), a single primitive wrench set is often sufficient for modelling the forces generated during the process.

Example 5. We illustrate our approach by a planar fixture. Fig. 9(a) shows a planar part of thickness $1 / 4$ inches fixtured by four fixels. The part is made of AISI 1040 steel, with $E=200 \times 10^{9} \mathrm{~Pa}, \sigma_{s}=413 \times 10^{6} \mathrm{~Pa}$ and $\nu=0.3$. The machining tasks involve removing $1 / 8$ of an inch using a side mill along the edges $A B, C D$ and $G H$ so that $A B$ is accurately parallel to $C D$ and $G H$. The cutting force is estimated at 341 Newtons [18], lying in a sector which moves along the edge being machined. The fixels have spherical tips of uniform radius 2 inches, and are placed on the edges $A C, B H$, and $E F$ (each of $E F$ 's endpoints contact a fixel). For demonstration we focus on the milling force acting on the edge $A B$, and consider the variation of the quality measure $Q$ as the fixels $F_{1}$ and $F_{2}$, aligned vertically, move along 
the edges $A C$ and $B H$. Preload the fixture, for all fixel arrangements, such that the maximum shear stress near the contacts is $70 \%$ of the steel's yield stress. Using the Hertz contact model and the procedure outlined in Section 2.2 and detailed in Ref. [9], we found that the fixture satisfies the contact maintainability conditions for all cutting force positions and directions if the $F_{1}$ and $F_{2}$ are placed such that $x \leqslant 3.68$ inches. As shown in Fig. 9(b), when $x$ decreases from this value, the quality measure, defined using the vertices $A$ and $B$ as critical points, assumes a monotonically decreasing value, indicating improvement of fixture quality. Thus, of the fixtures we consider, the best one is given by placing the fixels $F_{1}$ and $F_{2}$ at $A$ and $B$, respectively, with $Q=2.4$ microns. This agrees with our intuition that this fixture arrangement best restrains the displacement of the edge $A B$.

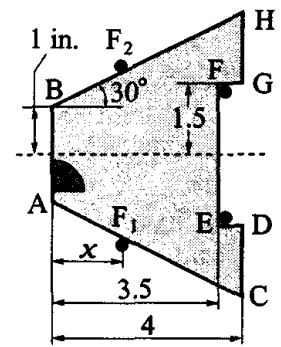

(a) A steel part fixture

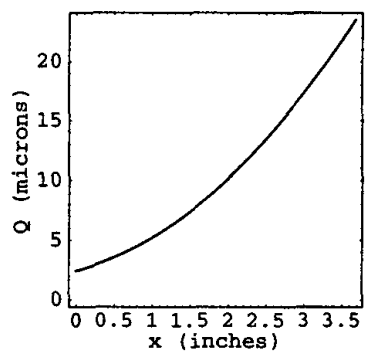

(b) Variation of the quality measure Fig. 9. Task primitive for milling operation

\section{Conclusion}

A task-dependent approach to minimum-deflection fixtures was presented. A notion of deflection for quasirigid bodies was formally defined, and was used to define a quality measure that characterizes the fixtured object's deflection with respect to given tasks. We also proposed a novel scheme for modelling task action by a class of primitive wrench sets. This scheme can be used to represent practical manufacturing operations, and provide an efficient structure for computing the quality measure. An example was finally used to illustrate that our approach can be effectively applied to compliant fixtures.

\section{References}

[1] D. P. Baron. Quadratic programming with quadratic constraints. Naval Research Logistics Quarterly, 19:253-260, 1972.

[2] R. C. Brost and R. R. Peters. Automatic design of 3d fixtures and assembly pallets. Technical Report SAND 95-2411, Sandia National Labs., 1997.

[3] J. P. Donoghue, W. S. Howard, and V. Kumar. Stable workpiece fixturing. In Proc. ASME Design Technical Conferences, 1994.

[4] C. Ferrari and J. Canny. Planning optimal grasps. In Proc. Int. Conf. on Robotics and Automation, pages 2290-2295, Nice, France, May 1992.
[5] H. Hanafusa and H. Asada. Stable prehension by a robot hand with elastic fingers. In Proc. $7^{\text {th }}$ Int. Symp. on Industrial Robots, pages 361-368. Reprinted in Robot Motion: Planning and Control, eds. M. Brady et al., 1977.

[6] W. S. Howard and V. Kumar. Stability of planar grasps. In Proc. Int. Conf. on Robotics and Automation, pages 28222827, 1994.

[7] D. G. Kirkpatrick, B. Mishra, and C. K. Yap. Quantitative steinitz's theorems with applications to multifingered grasping. In Proc. $20^{\text {th }}$ ACM Symp. on Theory of Computing, pages 341-351, Baltimore, MD, May 1990.

[8] Z. Li and S. Sastry. Task oriented optimal grasping by multifingered robot hands. In Proc. Int. Conf. on Robotics and Automation, pages 389-394, Raleigh, NC, April 1987.

[9] Q. Lin. Mechanics and Planning of Workpiece Fixturing and Robotic Grasping. PhD thesis, Dept. of Mechanical Engineering, California Inst. of Technology, May 1998.

[10] Q. Lin, J. W. Burdick, and E. Rimon. Computation and analysis of compliance in grasping and fixturing. In Proc. Int. Conf. on Robotics and Automation, pages 93-99, 1997.

[11] Q. Lin, J. W. Burdick, and E. Rimon. A quality measure for compliant grasps. In Proc. Int. Conf. on Robotics and Automation, pages 86-92, 1997.

[12] Q. Lin, J. W. Burdick, and E. Rimon. Minimum-deflection grasps and fixtures. In Proc. Int. Conf. on Robotics and Automation, pages 3322-3328, Leuven, Belgium, May 1998.

[13] X. Markenscoff and C. H. Papadimitriou. Optimum grip of a polygon. Int. J. of Robotics Research, 8(2):17-29, 1989.

[14] B. Mirtich and J. Canny. Easily computable optimum grasps in 2-D and 3-D. In Proc. Int. Conf. on Robotics and Automation, pages 739-747, 1994.

[15] R. M. Murray, Z. Li, and S. S. Sastry. A Mathematical Introduction to Robotic Manipulation. CRC Press, Boca Raton, Florida, 1994.

[16] A. W. Naylor and G. R. Sell. Linear Operator Theory in Engineering and Science. Springer-Verlag, 1982.

[17] V.-D. Nguyen. Constructing stable grasps. Int. J. of Robotics Research, 8(1):26-37, 1989.

[18] E. Oberg, F. D. Jones, H. L. Horton, and H. H. Ryffel. Machinery's Handbook. Industrial Press, 24th edition, 1992.

[19] E. Rimon and J. W. Burdick. Mobility of bodies in contactii: How forces are generated by curvature effects. In Proc. Int. Conf. on Robotics and Automation, pages 2336-2341, 1994.

[20] M. Teichmann. A grasp metric invariant under rigid motions. In Proc. Int. Conf. on Robotics and Automation, pages 21432148, 1996.

[21] S. P. Timoshenko and D. H. Young. Elements of Strength of Materials. Van Nostrand, New York, 5th edition, 1968. 